DESY 00-104

HU-EP-00/30

NIC/DESY-00-002

Bicocca-FT-00-12

\title{
Comparative Benchmarks of full QCD Algorithms
}

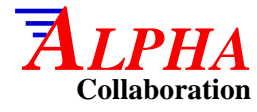 \\ Roberto Frezzotti \\ Dipt. di Fisica, Univ. di Milano Bicocca \\ Via Celoria 16, I-20133 Milano, Italy \\ Martin Hasenbusch, Ulli Wolff \\ Institut für Physik, Humboldt Universität \\ Invalidenstr. 110, D-10099 Berlin, Germany \\ Jochen Heitger \\ DESY \\ Platanenallee 6, D-15738 Zeuthen, Germany \\ Karl Jansen \\ CERN, Theory Division \\ CH-1211 Geneve 23, Switzerland
}

\begin{abstract}
We report performance benchmarks for several algorithms that we have used to simulate the Schrödinger functional with two flavors of dynamical quarks. They include hybrid and polynomial hybrid Monte Carlo with preconditioning. An appendix describes a method to deal with autocorrelations for nonlinear functions of primary observables as they are met here due to reweighting.
\end{abstract}




\section{Introduction}

In the past years the ALPHA Collaboration has pursued the goal to reliably compute the QCD gauge coupling at high energy in terms of non-perturbative low energy parameters. The concomitant necessity to deal with a large energy ratio in the continuum limit was solved by a breakup into recursive steps. Here one employs finite size rescaling by repeated factors of two and extrapolates to the continuum each step by itself. By a combination of theoretical reasoning and numerical tests the Schrödinger functional was determined as a particularly convenient framework for this purpose. The programme has been completed for the quenched approximation, see refs. [01, 2] for reviews of the approach and ref. [3] for a summary of data. First tests with a non vanishing flavour number have been reported [4]. As is well-known, by the inclusion of dynamical quarks the numerical cost is boosted by a large factor. The importance of algorithmic optimization can hence hardly be overestimated. The finite size technique with the Schrödinger functional - beside its uses for QCD physics - offers the possibility of an investigation of the lattice spacing dependence of the performance of fermion algorithms with all physical scales held fixed. Here we report on such results for several algorithms.

The Schrödinger functional can be regarded as the free energy $\Gamma$ of QCD in a finite volume $L^{3} \times T$,

$$
\exp (-\Gamma)=\int D[U] D[\bar{\psi}] D[\psi] \exp (-S[U, \bar{\psi}, \psi]) .
$$

The action $S$ consists of the usual plaquette action for SU(3) gauge fields $U$ and two degenerate flavours of clover-improved Wilson fermions. The box is periodic in space, and fixed gluon potentials and vanishing quark fields' are prescribed on the temporal boundaries. The boundary potentials $C$ and $C^{\prime}$ at $x_{0}=0$ and $x_{0}=T$ are specified in terms of the scale $L$ and dimensionless parameters, one of which is called $\eta$ and is kept variable. A convenient practical choice with $T=L$, introduced as point "A" in [5, is used throughout. Quark fields are periodic in space up to a phase $\theta=\pi / 5$. An Abelian background field is induced which can be varied by changing $\eta$. The response to such an infinitesimal variation is used to define the renormalized

\footnotetext{
${ }^{1}$ Non-vanishing quark sources are also possible but will not be needed here.
} 
coupling

$$
g_{\mathrm{SF}}^{2}(L)=\left.\frac{\partial \Gamma_{0} / \partial \eta}{\partial \Gamma / \partial \eta}\right|_{\eta=0},
$$

where $\Gamma_{0} / g_{0}^{2}$ is the tree level value of $\Gamma$ for bare coupling $g_{0}$. For a lattice realization we now have to choose values for $L / a, g_{0}$ and bare quark mass $m_{0}$ as well as coefficients for the improvement terms in the action. We take the latter as smooth functions of $g_{0}$ either by a perturbative expression or by a non-perturbative fit [6]. The mass $m_{0}$ is fixed by demanding zero PCAC-mass [7]. Hence we may approach the continuum limit by a sequence of lattices with growing $L / a$ and $g_{0}$ adjusted to maintain a fixed value $g_{\mathrm{SF}}$. Conceptually this is exactly the same situation as in our quenched computations. The regularizing lattice spacing $a$ varies while renormalized physics is held fixed. It is on such 'trajectories', that we study algorithm performance.

Our most extensive simulations of the $\mathrm{O}(a)$ improved Schrödinger functional have been conducted with the well-known hybrid Monte Carlo method (HMC) [8]. In our implementation we took advantage of preconditioning and the refinement proposed in [9]. It amounts to the introduction of two different step sizes for fermion-gluon and gluonic self-couplings in an approximately optimal proportion depending on their relative computational cost. In other long runs we applied the polynomial hybrid Monte Carlo (PHMC) [10, 11]. Here, as for the multiboson technique [12], an approximately inverting polynomial of the Dirac operator is used to bosonize the theory. In the multiboson proposal the resulting action is represented by many boson fields with nearest neighbour couplings. For unimproved Wilson fermions finite step-size updates are employed, which however become impractical when the clover term is included - the case on that we concentrate here. A further disadvantage is the additional slowing down due to collective effects of the many bosons [13]. With PHMC the operator polynomial is employed to construct a non-local Gaussian action for only one boson field which is simulated by HMC. The imperfection of the polynomial can be corrected by an acceptance or reweighting step. Some results are reported which have been obtained by a recently proposed multi-level Metropolis procedure (MLM) [14]. Further details of the various algorithms will be given below. 


\section{Algorithms in this study}

In this section we briefly describe our implementations of fermion Monte Carlo algorithms as they are benchmarked in this study. With each of them the goal is the inclusion of effects of the weight factor $\operatorname{det}(Q)^{2}$ which arises from integrating two degenerate flavours of quarks out of (1.1),

$$
\exp (-\Gamma)=\int D[U] \exp \left(-S_{\text {gauge }}[U]\right) \operatorname{det}(Q)^{2} .
$$

Here the hermitian operator $Q$ for Sheikholeslami-Wohlert improved Wilson quarks has the structure

$$
Q=c_{0} \gamma_{5} M ; \quad M=1-T-H .
$$

The constant $c_{0}$ is chosen to contain the eigenvalues of $Q$ in the interior of the interval $(-1,1)$. The matrix $M$ contains nearest neighbour hopping terms in $H$ and the clover term in $T$, which is diagonal with respect to the lattice index. The detailed form of these components, including boundary improvement, can for instance be found in ref. [15].

\subsection{Hybrid Monte Carlo}

The HMC method [8] has so far been the most popular fermion algorithm for QCD. In choosing a trajectory length of unity we followed the general experience that this is close to optimal. In [16] this was confirmed for the quenched Schrödinger functional, and a test with dynamical fermions at $L / a=8$ showed an almost doubling of computational costs for $g_{\mathrm{SF}}^{2}$ as we lowered the trajectory length to one half. We reduced discretization errors by the multiple time scale method proposed in ref. [9] taking the version given there in eq. (6.7) with $n=4$. A test of the performance gain of the above integration scheme in practical simulations was performed in [17], where it was demonstrated that a substantial gain is achieved as compared to a standard leap-frog integrator. The value of $n$ was not varied any further in this study.

As an essential sophistication we made use of two different forms of preconditioning. Both rely on our ability to factor out of $Q$ matrix factors which on the one hand are easy to invert and on the other hand capture a part of its spectral variation to leave us with a better conditioned remaining factor. For 
even-odd preconditioning we exhibit the block structure of $M$ with respect to even (e) and odd (o) lattice sites and factorize

$$
M=\left(\begin{array}{cc}
M_{\mathrm{ee}} & M_{\mathrm{eo}} \\
M_{\mathrm{oe}} & M_{\mathrm{oo}}
\end{array}\right)=\left(\begin{array}{cc}
M_{\mathrm{ee}} & 0 \\
M_{\mathrm{oe}} & 1
\end{array}\right) \times\left(\begin{array}{cc}
1 & M_{\mathrm{ee}}^{-1} M_{\mathrm{eo}} \\
0 & M_{\mathrm{oo}}-M_{\mathrm{oe}} M_{\mathrm{ee}}^{-1} M_{\mathrm{eo}}
\end{array}\right),
$$

where the left (lower) block-triangular factor and the block-diagonal $M_{\mathrm{ee}}$ are easy to invert. This factorization can now be used in a two-fold way. If the original $Q$ under the determinant in (2.1) is plugged into the HMC algorithm we have to continuously solve linear systems with coefficient matrices given by $Q$. With (2.3) these can be transformed into better conditioned systems with accelerated iterative inversion of

$$
\hat{Q}=\tilde{c}_{0} \gamma_{5}\left(M_{\mathrm{oo}}-M_{\mathrm{oe}} M_{\mathrm{ee}}^{-1} M_{\mathrm{eo}}\right) .
$$

The constant $\tilde{c}_{0}$ is again used to normalize the spectrum of $\hat{Q}$. On the other hand we may also conclude from (2.3) that up to irrelevant constant factors the relation

$$
\operatorname{det}(Q) \propto \operatorname{det}\left(M_{\mathrm{ee}}\right) \operatorname{det}(\hat{Q})
$$

holds. Now $\hat{Q}$ enters into the HMC and leads to a different Monte Carlo dynamics, which also takes $\operatorname{det}\left(M_{\mathrm{ee}}\right)$ into account. When we refer to evenodd preconditioning in this paper, this second variant will always be meant. Further details on our implementation of HMC may be found in [18]. As we only have to invert the squared operator $\hat{Q}^{2}$ we use the conjugate gradient method (CG), which was found to be close to optimal in this case.

For SSOR preconditioning a different factorization of $M$ based on factors triangular with respect to a lexicographic ordering of lattice sites is used [19, 20, 21]. Due to its complexity, in particular if the clover term is included, this has to our knowledge only been used to accelerate linear systems and was for that purpose reported to be superior over even-odd preconditioning if combined with the BiCGstab [22] inversion algorithm for the preconditioned $M$ and $M^{\dagger}$. In the following SSOR will refer to such an implementation. For the unimproved case, a simplified form of SSOR preconditioning (ILU) was implemented under the determinant with very positive results [10].

We compared the performance of our two HMC program versions on our largest lattice, i.e. $12^{4}$ at $\beta=9.5$. In solving the linear systems with the respective preconditioned operators we confirmed that in terms of operations associated with applying these operators to fields, the BiCGstab algorithm 
with SSOR preconditioning outperforms the CG algorithm with even-odd preconditioning by a factor of about 1.6. Part of this advantage is however lost in terms of CPU time, because on our Alenia Quadrics (APE) machines inner products are relatively expensive. Since in the BiCGstab algorithm inner products and linear combinations are much more frequent than in the CG algorithm, this is a non-negligible overhead. The overall advantage that we find for the even-odd version derives however from the different operators under the determinant. A clear sign of this is the behaviour of the acceptance rate in both cases. While for the even-odd preconditioned determinant we could obtain an acceptance rate of $91 \%$ with a step size of $\Delta \tau=0.08$, for $\operatorname{det}\left(Q^{2}\right)$ it went down to $75 \%$ already at a step size of $\Delta \tau=0.07$.

In principle, one could also conceive of the following combination yet untested by us. One uses the even-odd preconditioned determinant and, when linear systems with $\hat{Q}$ have to be solved, one transforms them to the SSOR preconditioned form, solves, and translates back. It is unclear at present, whether the overhead still leaves this variant profitable.

\subsection{Polynomial Hybrid Monte Carlo}

We recall here some basics of the PHMC algorithm. For technical details the reader is referred to refs. [23, 24]. In the PHMC algorithm the inverse of $\hat{Q}^{2}$ is approximately computed by a suitable [12] Chebyshev polynomial of degree $n$,

$$
\hat{Q}^{-2} \approx P_{n, \epsilon}\left(\hat{Q}^{2}\right) .
$$

Defining the relative deviation

$$
R_{n, \epsilon}(\lambda)=\lambda P_{n, \epsilon}(\lambda)-1,
$$

the inversion error for eigenvalues $\lambda \in[\epsilon, 1]$ of $\hat{Q}^{2}$ is bounded by

$$
\delta=\sup _{\lambda}\left|R_{n, \epsilon}(\lambda)\right|=2\left(\frac{1-\sqrt{\epsilon}}{1+\sqrt{\epsilon}}\right)^{n+1} .
$$

For a given degree $n$ the free parameter $\epsilon$ in $P_{n, \epsilon}$ allows to trade between approximation range and accuracy. For eigenvalues $\lambda<\epsilon$ the error monotonically moves from $R_{n, \epsilon}(\epsilon)=-\delta$ to $R_{n, \epsilon}(0)=-1$. With the help of $P_{n, \epsilon}$ we represent the determinant by a bosonic spinor field (pseudofermion) $\phi$

$$
\operatorname{det}\left(\hat{Q}^{2}\right)=\int D[\phi] D\left[\phi^{\dagger}\right] \exp \left(-S_{P}\right) W
$$


with the Gaussian action

$$
S_{P}=\phi^{\dagger} P_{n, \epsilon}\left(\hat{Q}^{2}[U]\right) \phi
$$

and the remainder

$$
W=\operatorname{det}\left(\hat{Q}^{2} P_{n, \epsilon}\left(\hat{Q}^{2}\right)\right)
$$

rendering (2.9) exact. As long as the spectrum of $\hat{Q}^{2}$ is in the approximation range $[\epsilon, 1], W$ is a small correction close to one. Expectation values in the full QCD ensemble are now given by reweighting with $W$ as

$$
\langle\mathcal{O}\rangle=\frac{\langle\mathcal{O} W\rangle_{P}}{\langle W\rangle_{P}}
$$

where $\mathcal{O}$ is some observable and the average $\langle\ldots\rangle_{P}$ is taken with the action $S_{\text {gauge }}+S_{P}$. Since $W$ is still given by a determinant, a straightforward evaluation is hard. As it is a small correction, however, stochastic (unbiased) estimates should be adequate. For each measurement we construct an estimator $\bar{W}$ given by

$$
\bar{W}=\frac{1}{N_{\text {corr }}} \sum_{i=1}^{N_{\text {corr }}} \exp \left\{\eta_{i}^{\dagger}\left(1-\left[\hat{Q}^{2} P_{n, \epsilon}\left(\hat{Q}^{2}\right)\right]^{-1}\right) \eta_{i}\right\}
$$

with independent Gaussian random fields $\eta_{i}$. Averaging over $N_{\text {corr }}$ such estimates allows us to reduce and control the extra noise inflicted here. The true QCD average is then estimated by eq. (2.12) with $W$ replaced by $\bar{W}$.

The update of the gauge field and the pseudofermionic field $\phi$ follows the standard HMC pattern with global heatbath for $\phi$ and molecular dynamics for $U$ including the speedup from [9] discussed before. This is chosen, because, in contrast to the multiboson algorithm [12], finite step size updates for $U$ are impractical here due to the complicated non-local effective action.

At this point the parameters $n, \epsilon$ and, less importantly, $N_{\text {corr }}$ are at our disposal for optimization. For small eigenvalues the growth of the 'inverter' $P_{n, \epsilon}(\lambda) \sim 1 / \lambda$ is cut off at $\lambda \sim \epsilon$. For the HMC dynamics $\epsilon$ hence, in some sense, takes over the role of the smallest eigenvalue. It was found advantageous [23, 24] to choose $\epsilon$ a few times larger than the typical smallest eigenvalue of $Q^{2}$. This allows us to keep the degree of the polynomial lower for the same approximation accuracy. Configurations with small eigenvalues of $\hat{Q}^{2}$ are produced more frequently than they would be with the exact 
determinantal weight. As the algorithm is still exact, this is precisely compensated by $W$ or respectively $\bar{W}$ giving smaller weight to the observables evaluated on these configurations. It should be borne in mind that the unquenched lattice path integral is always well-defined. The potential problem with nearly 'exceptional' configurations is a statistical one with rarely sampled large contributions, which is alleviated by our sampling and reweighting technique. This is the reason for us to prefer the reweighting correction over an acceptance step.

There is a special round-off problem for PHMC that we briefly summarize now with more details available in [23, 25]. To generate $\phi$ with action (2.10) it is necessary to factorize

$$
P_{n, \epsilon}\left(\hat{Q}^{2}\right)=F_{n}(\hat{Q})^{\dagger} F_{n}(\hat{Q})
$$

with an $n$ 'th degree polynomial $F_{n}$. For gauge field updating $U$-derivatives of $S_{P}$ have to be taken at fixed $\phi$. To this end we factorize further

$$
F_{n}(\hat{Q}) \phi=\left[\sqrt{c_{n}}\left(\hat{Q}-r_{n}\right)\right]\left[\sqrt{c_{n-1}}\left(\hat{Q}-r_{n-1}\right)\right] \cdots\left[\sqrt{c_{1}}\left(\hat{Q}-r_{1}\right)\right] \phi
$$

and store the occurring subproducts to facilitate the force computation. While the complex roots $r_{k}$ are determined by $P_{n, \epsilon}$, the real factors $\sqrt{c_{k}}$ only serve to prevent the partial products from growing too large or too small. It is known [25] that the evaluation of a high order matrix polynomial in factorized form is in principle rather susceptible to round-off error. In particular, the ordering of factors in (2.15) is of crucial importance in this context. In [23, 25] orderings were found which make this source of errors negligible for the runs with $n$ up to 46 reported in this paper, even on the 32-bit APE 100 machines. Further details on tuning the polynomial parameters are deferred to appendix B.

Another variant of PHMC could be devised by applying an inverting polynomial to the complex spectrum of $\gamma_{5} \hat{Q}$ instead of the real positive $\hat{Q}^{2}$. A corresponding multiboson algorithm was investigated in ref. [26]. We shall investigate this method in the near future.

\subsection{Multi-Level Metropolis Algorithm}

As for the previous algorithms it is our aim to represent $\operatorname{det}\left(Q^{2}\right) \propto \operatorname{det}\left(M^{\dagger} M\right)$ in a way suitable for simulation. Here this will be done in part by the explicit use of a few terms of the hopping parameter expansion in powers of $T+H$ 
(see eq. (2.2)) and by integrals over a collection of pseudofermion fields to represent the remainder.

The series for the logarithm of the determinant is given by

$$
\log \operatorname{det}(M)=\operatorname{tr} \ln (M)=-\operatorname{tr}(T+H)-\frac{1}{2} \operatorname{tr}(T+H)^{2}-\frac{1}{3} \operatorname{tr}(T+H)^{3}+\ldots
$$

In our algorithm, we separate off the series up to some order $k$. In the present work we found it convenient to use $k=3$, since $\operatorname{tr}(T+H)^{i}=\operatorname{tr} T^{i}$ can easily be computed for $i=1,2,3$. At higher orders also mixed terms would contribute. In order to deal with the remaining terms, we define

$$
\tilde{M}=M \exp \left(\sum_{j=1}^{k} \frac{1}{j}(T+H)^{j}\right) .
$$

For the inverse of $\tilde{M}$ we introduce a hierarchical approximation by polynomials $P_{i}$ of order $n_{i}$ in $T+H$,

$$
\tilde{M}^{-1}=\prod_{i=1}^{j} P_{i}^{r_{i}}+\mathrm{O}\left((T+H)^{n_{j}+1}\right)
$$

The full required inversion accuracy is reached for the maximal value $j=$ $l$. This is used with $r_{1}+r_{2}+\ldots+r_{l}$ pseudofermion fields to derive the representation

$$
\operatorname{det}\left(\tilde{M}^{\dagger} \tilde{M}\right) \propto \int \prod_{i=1}^{l} \prod_{a=1}^{r_{i}} D\left[\phi_{i a}^{\dagger}\right] D\left[\phi_{i a}\right] \exp \left(-\sum_{i, a}\left|P_{i} \phi_{i a}\right|^{2}\right) .
$$

The powers $r_{i}>1$ are advantageous as they lead to a smaller force on the gauge field which allows larger update steps [14.

Let us summarize the action that is simulated,

$$
S=S_{\text {gauge }}[U]+S_{\text {hop }}[U]+S_{\mathrm{PF}}[U, \phi]
$$

with'

$$
S_{\text {hop }}[U]=\operatorname{tr} T+\frac{1}{2} \operatorname{tr} T^{2}+\frac{1}{3} \operatorname{tr} T^{3}
$$

\footnotetext{
${ }^{2} \operatorname{tr} T$ is small but nonzero, as we also included boundary improvement terms in it.
} 
and

$$
S_{\mathrm{PF}}[U, \phi]=\sum_{i, a}\left|P_{i} \phi_{i a}\right|^{2} .
$$

We now consider $S_{\text {gauge }}[U]$ as a zeroth approximation which is taken into account in generating finite stepsize primary update proposals. All further terms will eventually be implemented by accept-reject filters. In the first level action

$$
S^{(1)}=S_{\text {gauge }}+S_{\mathrm{hop}}+\sum_{a=1}^{r_{1}}\left|P_{1} \phi_{1 a}\right|^{2}
$$

the hopping term is included as it would otherwise lead to a single link action too complex for level zero. All further levels $j>1$ are straightforwardly given by

$$
S^{(j)}=S^{(j-1)}+\sum_{a=1}^{r_{j}}\left|P_{j} \phi_{j a}\right|^{2}
$$

up to $j=l$.

We now describe the steps of the multi-level Metropolis update scheme. A proposal at level 0 is given by multiple local updates with the CabibboMarinari heatbath or the overrelaxation algorithm. One possibility is to randomly select $v$ links to be updated. In ref. [14] we found it advantageous to actually update several times a sublattice chosen at random from a set that covers the lattice. The size of the sublattices is chosen such that a reasonable acceptance at level one of the algorithm is achieved. The whole proposal obeys detailed balance with respect to the level zero action as we reverse the order of link updates with probability $1 / 2$. The remaining levels now proceed recursively as follows. Generate a proposal $\tilde{U}$ by performing $t_{j}$ update steps at level $j-1$. Accept $\tilde{U}$ as new configuration at level $j$ with the probability

$$
A^{(j)}=\min \left[1, \exp \left(-\Delta S^{(j)}[\tilde{U}]+\Delta S^{(j)}[U]\right)\right],
$$

where $\Delta S^{(j)}=S^{(j)}-S^{(j-1)}$.

In our implementation, the auxiliary fields $\phi_{i a}$ are kept fixed during the update cycle of the gauge field after they have been generated by a global heatbath by solving

$$
\phi_{i, a}=P_{i}^{-1} \eta,
$$

where $\eta$ is a Gaussian random field. 
In its present form the MLM algorithm will not be the method of choice for large lattices. The reason is that its cost will ultimately grow proportional to the square of the number of lattice sites. This is because the size of the updated blocks cannot grow while maintaining the acceptance rate and thus their number is proportional to the volume. Each evaluation of $\Delta S^{(j)}$ is however also of order volume in complexity. On the other hand, as we shall see shortly, MLM can produce precise results at $L / a=5$ where we have tested it here. As it contains interesting elements, for instance being a finite step-size method for improved dynamical fermions, we still found the idea and the practical test worth reporting and comparing with other methods here, for instance as a basis for further modification.

\section{Benchmarks of algorithmic performance}

\subsection{Our measure of efficiency}

We now define two quantities, $M_{\text {cost }}$ and $D_{\text {cost }}$, which allow to compare simulation costs for a certain physics output between different algorithms and parameters. The first measure is machine dependent and refers to actual CPU time on the APE100 line of parallel computers currently in use by the ALPHA Collaboration. The second one is machine independent with the number of Dirac operator applications to a spinor field being our currency. As a target quantity, whose statistical accuracy is used for weighing costs in either units, we take our coupling $g_{\mathrm{SF}}^{2}$. The precise definitions are

$$
\begin{aligned}
M_{\text {cost }}= & (\text { update time in seconds on machine M) } \\
& \times\left(\text { error of } 1 / g_{\mathrm{SF}}^{2}\right)^{2} \times(4 a / T)(4 a / L)^{3}
\end{aligned}
$$

and

$$
D_{\text {cost }}=(\text { number of applications } Q \phi) \times\left(\text { error of } 1 / g_{\mathrm{SF}}^{2}\right)^{2} \text {. }
$$

Since the squared error in both formulas goes down inversely proportional to the run length, both quantities, extracted from given Monte Carlo simulations, do not depend on their lengths. The reason for focusing on absolute errors of $1 / g_{\mathrm{SF}}^{2}$ is as follows. We assume for the purpose of error analysis only that the running of $g_{\mathrm{SF}}$ with $L$ has the structure of 1-loop perturbation theory, $1 / g_{\mathrm{SF}}^{2} \approx-2 b_{0} \log L+$ const. Then

$$
\frac{\delta L}{L}=\frac{1}{2 b_{0}} \delta\left(1 / g_{\mathrm{SF}}^{2}\right) \approx 8 \delta\left(1 / g_{\mathrm{SF}}^{2}\right)
$$


holds, and we approximately aim at a certain relative scale uncertainty, independently of the size of $g_{\mathrm{SF}}$. In (3.1) the trivial growth proportional to the number of lattice sites is cancelled such that both quantities scale in the same way. The reference machine $\mathrm{M}$ in this publication will always be the smallest 8-node machine of type Q1. Most of our data actually come from bigger machines with up to 512 nodes. The costs on these machines are converted by multiplying naively by the ratio of nodes, e.g. 512/8. This means, we neglect communication overheads, which is a small effect on our hardware and implementation. Note that with our definitions costs can be meaningfully compared also under trivial (replica) parallelization, of which we make extensive use.

While most of the CPU time with dynamical fermions is spent on applications of the Dirac operator there is also quite some overhead from other operations, in particular on small lattices. This was neglected in $D_{\text {cost }}$ except for the contribution to the gauge field force in the PHMC algorithm which is proportional to the polynomial degree $n$. As a consequence, the ratio $D_{\text {cost }} / M_{\text {cost }}$ varies between $50 \%$ and $80 \%$ of the theoretical value referring to $Q \phi$ operations only. Applications of $Q$ and $\hat{Q}$ are so close to each other both in theoretical complexity and actual CPU time, that we neglect their difference. The SSOR preconditioned operator, on the other hand, is counted as $4 / 3 Q$ operations due to extra multiplications of the diagonal (clover) part.

A typical run that entered our benchmarks is entry $12 \mathrm{k}$ in Table 1 . With $M_{\text {cost }} \approx 12$ and $D_{\text {cost }} \approx 3000$ we ran a total of 13000 trajectories on an $L / a=T / a=12$ lattice and achieved $6 \%$ scale accuracy in about 6 days on 256 nodes of APE100.

\subsection{Numerical results}

In Table 1 we list the most important parameters for a subset of our HMC and PHMC runs performed to investigate the QCD running gauge coupling with two massless flavours?. The fourth column indicates which of the algorithms discussed before was used. This table has to be read in conjunction with Table 2, where the measured values for $M_{\text {cost }}$ and $D_{\text {cost }}$ are given. Results for $D_{\text {cost }}$ of MLM follow in Table 4 below. Error estimates for the costs stem from the error of $\tau_{\mathrm{int}}$ of $g_{\mathrm{SF}}^{-2}$ which is determined by the method of

\footnotetext{
${ }^{3}$ The physical implications of these data will be analyzed in a separate paper [29] while here we focus on algorithmic aspects.
} 
Appendix A. Its value here refers to a unit given by complete update cycles (trajectories). The update time in seconds for one such cycle is normalized to the APE100-Q1 as discussed with the definition of $M_{\text {cost }}$. In Fig. 1 we plot $M_{\text {cost }}$ against $L / a$ for all our runs at a fixed scale $L$ in physical units that is implicitly determined by the condition $g_{\mathrm{SF}}^{2} \approx 1.1$. The line corresponding to a growth proportional to $a^{-3}$ is shown as a reference and roughly represents the rise of the data. This combines effects of a growing variance of our observable in the Schrödinger functional for $g_{\mathrm{SF}}^{2}$ and of critical slowing down. The latter accounts for about two powers of $1 / a$. In Fig. 2 the number of conjugate gradient iterations is shown for our even-odd preconditioned HMC runs. At least for smaller $g_{\mathrm{SF}}^{2}$ there is an approximately linear growth with $L / a$ which contributes one power to critical slowing down. This is the expected behaviour since $1 / L$ is the infrared cutoff here analogous to the quark mass in other applications. At larger coupling $N_{\mathrm{CG}}$ moderately rises in the range that has been explored here.

The actual cost to determine the running coupling at fixed error for $g_{\mathrm{SF}}^{-2}$ as discussed before hence seems to roughly grow like $1 / a^{7}$ in the continuum limit, at least at the relatively weak coupling considered here. This seems to be more optimistic than the quark mass dependence in some previous estimates, for instance in [27]. One reason may be that our growth may be slightly underestimated due to overhead on the small lattices. Closer inspection reveals as another source of difference that in our molecular dynamics steps we are not forced to lower the step size $\Delta \tau$ at the rate usually estimated for constant acceptance while lowering the quark mass. Keeping $L$ however fixed in physical units means that $\beta$ rises when $a / L$ becomes smaller which makes the gauge field smoother at the same time. This could lead to smaller discretization errors and partly be responsible for the observed behaviour. The integration method [9] may in addition interfere with standard scaling on intermediate size lattices.

The vertical dotted line in Fig. 1 is located at $L / a=16$ and points to $M_{\text {cost }} \approx 30$. This implies about 100 days on 512 nodes for $3 \%$ scale accuracy. Thus at least with the next generation of APE1000 machines a serious continuum calculation should be within reach. For $L / a=12$, our most expensive lattices so far, we found a slight preference for PHMC. This conclusion holds also among the larger couplings simulated, with the costs being approximately independent of $g_{\mathrm{SF}}^{2}$ in the present range between 1.1

\footnotetext{
${ }^{4}$ See also the discussion in [28].
} 
and 1.8. All runs with $L / a=10$ have been performed employing HMC with SSOR 7 and reach up to much larger couplings. Here the growth of $M_{\text {cost }}$ and $D_{\text {cost }}$ with $g_{\mathrm{SF}}^{2}$ becomes clearly visible but not dramatic, see Fig. 3.

With the MLM algorithm for improved fermions we only have results for $L / a=5$ whose parameters are collected in Table 3 . As updates on level zero (gauge action only) we used heatbath sweeps and overrelaxation sweeps over certain sets of links. With probability $1 / 2$ we update either set A or set B. Set A consists of all spatial links of one randomly selected timeslice together with the temporal links at this timeslice in either positive or negative time direction. Set B are all links with a randomly selected spatial direction plus the temporal boundary links. In the case of set $\mathrm{A}$ we perform a heatbath sweep over all links followed by five overrelaxation sweeps. In the case of set $\mathrm{B}$ we perform a heatbath sweep over the temporal boundary links followed by five overrelaxation sweeps over the spatial links of one direction. In both cases the order of the updating is exactly reversed with probability $1 / 2$ to fulfill detailed balance. Given the large set of free parameters, some of them had to be chosen rather ad hoc. As we implemented and ran MLM on PCs but

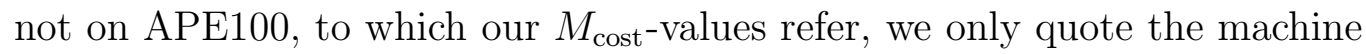
independent $D_{\text {cost }}$ here. Together with results for the coupling, which were found consistent with HMC results, they are given in Table 4.

\section{Conclusions}

We have studied several simulation algorithms for the Schrödinger functional of full QCD with two flavours of massless quarks. Due to relatively high statistics on lattices up to $12^{4}$ we obtained precise information on integrated autocorrelation times. Although our results are relatively close for the algorithms compared, there is a slight advantage for the polynomial hybrid Monte Carlo for our parameter range and observable. For this numerical reason and for the expected advantages from its modified sampling at larger coupling, we plan to focus on PHMC for our coming runs with $L / a=16$. In simulations with ordinary HMC we found even-odd preconditioning of the determinant more efficient than SSOR preconditioning the solver alone.

Acknowledgement We would like to thank Rainer Sommer for numerous discussions and for a critical reading of the manuscript. We are grateful to

\footnotetext{
${ }^{5}$ Our even-odd preconditioned (P)HMC code is unsuitable for this lattice size due to machine topology.
} 
DESY for allocating computer time on the APE machines at DESY Zeuthen.

\section{A Integrated autocorrelation time for func- tions of observables}

In this appendix we discuss a method to assess the effect of autocorrelations on the statistical error of nonlinear functions of simple expectation values. We consider a number of observables in a statistical system, and by $A_{\alpha}, \alpha=1,2, \ldots$ we denote their exact mean values. For each observable we have a chain of $N$ unbiased but (auto-)correlated Monte Carlo estimates $a_{\alpha}^{i}, i=1, \ldots, N$. Assume that we want to estimate $F=f\left(A_{\alpha}\right)$, where $f$ is an in principle arbitrary function. A simple case arising in the context of reweighting is the quotient $F=A_{1} / A_{2}$, while fit parameters extracted from a correlation function at a sequence of separations would be a more complicated case.

The obvious estimator for $F$ is given by $f\left(\bar{a}_{\alpha}\right)$, where

$$
\bar{a}_{\alpha}=\frac{1}{N} \sum_{i=1}^{N} a_{\alpha}^{i}
$$

are the ensemble-means for our simulation. In a correct and equilibrated Monte Carlo we expect

$$
\begin{aligned}
\left\langle A_{\alpha}-\bar{a}_{\alpha}\right\rangle & =0 \\
\left\langle\left(A_{\alpha}-\bar{a}_{\alpha}\right)^{2}\right\rangle & =\mathrm{O}(1 / N)
\end{aligned}
$$

to hold, where the expectation values in this appendix mean the average over an infinite number of identical Monte Carlo simulations of length $N$. Loosely speaking, $A_{\alpha}$ and $\bar{a}_{\alpha}$ differ by $\mathrm{O}(1 / \sqrt{N})$ in an individual Monte Carlo run.

By Taylor expanding $f$ around the argument $A_{\alpha}$ we find

$$
\begin{aligned}
\left\langle F-f\left(\bar{a}_{\alpha}\right)\right\rangle & =\mathrm{O}(1 / N), \\
\sigma^{2}=\left\langle\left(F-f\left(\bar{a}_{\alpha}\right)\right)^{2}\right\rangle & =\mathrm{O}(1 / N) .
\end{aligned}
$$

The first line reveals the (in general unavoidable) bias of our estimator which has to be suppressed by large enough $N$. The statistical error $\sigma$ of order

\footnotetext{
${ }^{6}$ In principle it is also possible to cancel the leading bias-term, for instance by the jackknife method.
} 
$1 / \sqrt{N}$ will be discussed in the following. With the gradient vectors

$$
H_{\beta}=f_{\mid \beta}\left(A_{\alpha}\right), \quad \bar{h}_{\beta}=f_{\mid \beta}\left(\bar{a}_{\alpha}\right)
$$

we define projected observables

$$
A_{H}=\sum_{\alpha} A_{\alpha} H_{\alpha}
$$

and analogously with $\bar{h}_{\alpha}$ and for $\bar{a}_{\alpha}$. Now we conclude that up to higher orders in $1 / N$ we just need to know the variance of the projected observable,

$$
\sigma^{2} \approx\left\langle\left(A_{H}-\bar{a}_{H}\right)^{2}\right\rangle \approx\left\langle\left(A_{\bar{h}}-\bar{a}_{\bar{h}}\right)^{2}\right\rangle .
$$

In practice, the projection can only be performed with $\bar{h}$, taken from the data, of course.

From here on one may proceed just like in the case of simple expectation values. We may estimate the relevant autocorrelation function at separation $t$ for instance】 as

$$
\Gamma(t)=\frac{1}{N-t} \sum_{i=1}^{N-t}\left(a_{\bar{h}}^{i}-\frac{1}{N-t} \sum_{j=1}^{N-t} a_{\bar{h}}^{j}\right)\left(a_{\bar{h}}^{(i+t)}-\frac{1}{N-t} \sum_{k=t+1}^{N} a_{\bar{h}}^{k}\right) .
$$

From it the error follows as

$$
\sigma^{2}=\frac{\Gamma(0)}{N} 2 \tau_{i n t}
$$

with

$$
\tau_{\text {int }}=\frac{1}{2}+\sum_{t=1}^{W} \frac{\Gamma(t)}{\Gamma(0)} .
$$

The summation window $W$ is usually chosen large enough that $\tau_{\text {int }}$ saturates to a constant within statistical errors. Often this can be achieved by selfconsistently summing until $W / \tau_{\text {int }}$ reaches numbers like $5 \ldots 10$. Below the role of $W$ will be discussed further. In summary, the deviation of $2 \tau_{i n t}$ from one for the projected observable describes the complete effect of (auto)correlations on the estimation of $F$. Obviously, $\sigma, \Gamma$ and $\tau_{\text {int }}$ all depend on the function $f\left(A_{\alpha}\right)$ which has remained implicit in our notation.

\footnotetext{
${ }^{7}$ Less symmetrically, one might also subtract $\bar{a}_{\bar{h}}$ in each bracket.
} 
We would like to conclude this appendix by indicating the advantage of explicitly summing $\Gamma$ compared to the jackknife binning procedure which is often applied for the estimation of errors of secondary quantities like bestfit parameters. There one divides $N$ estimates into $N / B$ bins of length $B$. The individual bins are treated as uncorrelated. The fact that this is not exactly true leads to a systematic error in the error estimate which is of order $\tau / B$ from the correlation of neighbouring bins. Here $\tau$ is a general scale of autocorrelation times involved. This is usually controlled by demanding a plateau of the errors as the bin length is varied. The statistical uncertainty of the error estimate is of order $\sqrt{B / N}$. These two errors have to be balanced at an optimal bin length, which incidentally scales as $B \propto\left(N \tau^{2}\right)^{1 / 3}$.

If we sum $\Gamma$ up to separation $W$ (summation window), systematic errors due to the neglected remainder are of order $\exp (-W / \tau)$. The statistical error of the error estimate is expected to be of order $\sqrt{W / N}$ from the number of independent windows. In fact, Madras and Sokal quote the formula [30]

$$
\frac{\delta \tau_{\text {int }}}{\tau_{\text {int }}}=\sqrt{\frac{2(2 W+1)}{N}}
$$

which follows if one approximates the required summed 4-point autocorrelation function by its disconnected part which falls back to the sum over $\Gamma$ entering into $\tau_{\text {int }}$ itself. In practice, these errors usually look very reasonable under repeated runs. The conclusion is that the systematic errors for the "summation method" are much smaller, which, in balancing systematic with statistical errors, leads to more accurate error estimates. Taking the idea of balancing totally seriously, one would conclude that the "error of the error" decays like $[1 / N]^{1 / 3}$ with binning and with $[\ln (N) / N]^{1 / 2}$ with the $\Gamma$-summation method.

\section{B Tuning of the PHMC algorithm}

Here we summarize our strategy for tuning the free parameters of the PHMC algorithm, in particular for the Schrödinger functional at small volume or weak coupling. We are interested in the error $\sigma_{N_{\text {corr }}}$ of the estimate

$$
\langle\mathcal{O}\rangle=\frac{\langle\mathcal{O} \bar{W}\rangle_{P}}{\langle\bar{W}\rangle_{P}},
$$


for some observable $\mathcal{O}$ (mainly $g_{\mathrm{SF}}^{-2}$ at present) and $\bar{W}$ given in eq. (2.13). All errors are assessed by the method of the previous appendix. Here the error can be decomposed as

$$
\sigma_{N_{\text {corr }}}^{2}=\sigma_{P}^{2}+\left[\sigma_{\infty}^{2}-\sigma_{P}^{2}\right]+\left[\sigma_{N_{\text {corr }}}^{2}-\sigma_{\infty}^{2}\right] .
$$

Here $\sigma_{\infty}^{2}$ corresponds to $N_{\text {corr }}=\infty$, or equivalently to the use of the exact reweighting with $W$ as in eq. (2.12), while $\sigma_{P}^{2}$ refers to the simple mean $\langle\mathcal{O}\rangle_{P}$. The second term in (B.2) reflects a contribution from "ideal" reweighting and hence from the imperfection of the polynomial approximation, while the third one is due to our non-ideal stochastic estimation of the correction. Both would vanish for a perfect polynomial and are naively proportional to $\delta^{2}$, the scale of polynomial errors. The third term has a factor $1 / N_{\text {corr }}$ in addition. We can roughly disentangle them by measuring $\langle\mathcal{O}\rangle_{P}$ and $\langle\mathcal{O}\rangle$ and their errors for at least two values of $N_{\text {corr }}$. The goal now is to take $N_{\text {corr }}=1 \ldots 4$ and find $\delta, \epsilon$ such that the reweighting part of the error remains acceptable, less than $20 \%$, say. This is to be achieved at the smallest possible value of the polynomial degree $n$.

In [24] we found for $\beta$ between 5.4 and 6.8 on $8^{3} \times 16$ the rule $\delta \approx 0.01$ and $\epsilon \approx 2 \lambda_{\min }$ to be very efficient, where $\lambda_{\min }$ is the average smallest eigenvalue of $\hat{Q}^{2}$. At the larger $\beta$-values of the present study we expect the intrinsic fluctuations caused by the gauge field to be smaller and correspondingly found a too large relative contribution from the third term in $(\mathbb{B} .2)$ when the above tuning is employed. Instead we found it much more efficient to attenuate this term with a smaller $\delta$. This turned out to be possible essentially without enlarging $n$, i.e. we could allow $\epsilon$ to grow even larger than $2 \lambda_{\min }$. This is probably due to smaller fluctuations of the small eigenvalues as well. In Table 5 and 6 we report simulation parameters of the PHMC runs on $12^{4}$. As to the choice of $\tilde{c}_{0}$ it is noted that one has to ensure $\lambda_{\max }<1$ for all configurations for a numerically stable evaluation of (2.15)). On the other hand, the efficiency is not very sensitive to the precise value of $\left\langle\lambda_{\max }\right\rangle$ which we hence kept safely below one. 


\section{References}

[1] M. Lüscher, Lectures given at Les Houches Summer School 1997, heplat/9802029.

[2] R. Sommer [ALPHA Collaboration], Lectures given at 36. Internationale Universitätswochen für Kern- und Teilchenphysik, Schladming 1997, hep-ph/9712218.

[3] S. Capitani, M. Lüscher, R. Sommer and H. Wittig [ALPHA Collaboration], Nucl. Phys. B544, 669 (1999) [hep-lat/9810063].

[4] J. Rolf and U. Wolff, Nucl. Phys. Proc. Suppl. 83-84, 899 (2000) [heplat/9907007].

[5] M. Lüscher, R. Sommer, P. Weisz and U. Wolff, Nucl. Phys. B413, 481 (1994) [hep-lat/9309005].

[6] K. Jansen and R. Sommer [ALPHA Collaboration], Nucl. Phys. B530, 185 (1998) [hep-lat/9803017].

[7] M. Lüscher, S. Sint, R. Sommer and P. Weisz, Nucl. Phys. B478, 365 (1996) [hep-lat/9605038].

[8] S. Duane, A. D. Kennedy, B. J. Pendleton and D. Roweth, Phys. Lett. B195, 216 (1987).

[9] J. C. Sexton and D. H. Weingarten, Nucl. Phys. B380, 665 (1992).

[10] P. de Forcrand and T. Takaishi, Nucl. Phys. Proc. Suppl. 53, 968 (1997) [hep-lat/9608093].

[11] R. Frezzotti and K. Jansen, Phys. Lett. B402, 328 (1997) [heplat/9702016].

[12] M. Lüscher, Nucl. Phys. B418, 637 (1994) [hep-lat/9311007].

[13] B. Jegerlehner, Nucl. Phys. B465, 487 (1996) [hep-lat/9512001].

[14] M. Hasenbusch, Phys. Rev. D59, 054505 (1999) [hep-lat/9807031].

[15] M. Lüscher and P. Weisz, Nucl. Phys. B479, 429 (1996) [heplat/9606016]. 
[16] B. Gehrmann and U. Wolff, Nucl. Phys. Proc. Suppl. 83-84, 801 (2000) [hep-lat/9908003].

[17] K. Jansen and C. Liu, Nucl. Phys. B453, 375 (1995) [hep-lat/9506020].

[18] K. Jansen and C. Liu, Comput. Phys. Commun. 99, 221 (1997) [heplat/9603008].

[19] S. Fischer, A. Frommer, U. Glassner, T. Lippert, G. Ritzenhofer and K. Schilling, Comput. Phys. Commun. 98, 20 (1996) [hep-lat/9602019].

[20] N. Eicker, W. Bietenholz, A. Frommer, H. Hoeber, T. Lippert and K. Schilling, Nucl. Phys. Proc. Suppl. 63, 955 (1998) [hep-lat/9709143].

[21] M. Guagnelli and J. Heitger [ALPHA Collaboration], Comput. Phys. Commun. 130, 12 (2000) [hep-lat/9910024].

[22] A. Frommer, V. Hannemann, B. Nockel, T. Lippert and K. Schilling, Int. J. Mod. Phys. C5, 1073 (1994) [hep-lat/9404013].

[23] R. Frezzotti and K. Jansen, Nucl. Phys. B555, 395 (1999) [heplat/9808011].

[24] R. Frezzotti and K. Jansen, Nucl. Phys. B555, 432 (1999) [heplat/9808038].

[25] B. Bunk, S. Elser, R. Frezzotti and K. Jansen, Comput. Phys. Commun. 118, 95 (1999) [hep-lat/9805026].

[26] A. Borrelli, P. de Forcrand and A. Galli, Nucl. Phys. B477, 809 (1996) [hep-lat/9602016].

[27] R. Gupta, A. Patel, C. F. Baillie, G. Guralnik, G. W. Kilcup and S. R. Sharpe, Phys. Rev. D40, 2072 (1989).

[28] K. Jansen, Nucl. Phys. Proc. Suppl. 53, 127 (1997) [hep-lat/9607051].

[29] ALPHA Collaboration, in preparation.

[30] N. Madras and A. D. Sokal, J. Stat. Phys. 21, 109 (1988). 


\begin{tabular}{|c|c|c|c|c|c|}
\hline set & $L / a$ & $\beta$ & algorithm & $\Delta \tau$ & $P_{\mathrm{acc}}$ \\
\hline $12 \mathrm{a}$ & 12 & 9.5 & HMC (e/o) & 0.080 & 0.91 \\
\hline $12 \mathrm{~b}$ & 12 & 9.5 & HMC (SSOR) & 0.060 & 0.86 \\
\hline $12 \mathrm{c}$ & 12 & 9.5 & HMC (SSOR) & 0.070 & 0.75 \\
\hline $12 \mathrm{~d}$ & 12 & 9.5 & PHMC (e/o) & 0.091 & 0.83 \\
\hline $12 \mathrm{e}$ & 12 & 9.5 & PHMC (e/o) & 0.100 & 0.76 \\
\hline $12 \mathrm{f}$ & 12 & 8.5 & HMC (e/o) & 0.070 & 0.93 \\
\hline $12 \mathrm{~g}$ & 12 & 8.5 & PHMC (e/o) & 0.091 & 0.80 \\
\hline $12 \mathrm{~h}$ & 12 & 8.5 & PHMC (e/o) & 0.114 & 0.75 \\
\hline $12 \mathrm{i}$ & 12 & 7.5 & $\mathrm{HMC}(\mathrm{e} / \mathrm{o})$ & 0.075 & 0.89 \\
\hline $12 \mathrm{j}$ & 12 & 7.5 & PHMC (e/o) & 0.098 & 0.77 \\
\hline $12 \mathrm{k}$ & 12 & 7.5 & PHMC (e/o) & 0.098 & 0.78 \\
\hline $10 \mathrm{a}$ & 10 & 9.3884 & HMC (SSOR) & 0.080 & 0.78 \\
\hline $10 \mathrm{~b}$ & 10 & 8.39 & HMC (SSOR) & 0.080 & 0.75 \\
\hline $10 \mathrm{c}$ & 10 & 7.3619 & HMC (SSOR) & 0.070 & 0.79 \\
\hline $10 \mathrm{~d}$ & 10 & 6.877 & HMC (SSOR) & 0.070 & 0.72 \\
\hline $10 \mathrm{e}$ & 10 & 6.5 & HMC (SSOR) & 0.060 & 0.80 \\
\hline $10 f$ & 10 & 6.0 & HMC (SSOR) & 0.050 & 0.83 \\
\hline $10 \mathrm{~g}$ & 10 & 5.5 & HMC (SSOR) & 0.040 & 0.82 \\
\hline $8 a$ & 8 & 9.2364 & $\mathrm{HMC}(\mathrm{e} / \mathrm{o})$ & 0.080 & 0.96 \\
\hline $8 b$ & 8 & 8.2373 & $\mathrm{HMC}(\mathrm{e} / \mathrm{o})$ & 0.120 & 0.91 \\
\hline $8 c$ & 8 & 7.2103 & HMC (SSOR) & 0.100 & 0.71 \\
\hline $6 a$ & 6 & 9.5 & HMC (e/o) & 0.110 & 0.97 \\
\hline $6 b$ & 6 & 9.0 & $\mathrm{HMC}(\mathrm{e} / \mathrm{o})$ & 0.110 & 0.97 \\
\hline $6 c$ & 6 & 8.5 & $\mathrm{HMC}(\mathrm{e} / \mathrm{o})$ & 0.100 & 0.97 \\
\hline $6 \mathrm{~d}$ & 6 & 7.5 & $\mathrm{HMC}(\mathrm{e} / \mathrm{o})$ & 0.070 & 0.98 \\
\hline $5 \mathrm{a}$ & 5 & 9.3884 & HMC (SSOR) & 0.120 & 0.92 \\
\hline $5 b$ & 5 & 7.3619 & HMC (SSOR) & 0.120 & 0.90 \\
\hline $4 a$ & 4 & 9.2364 & HMC (e/o) & 0.130 & 0.98 \\
\hline $4 b$ & 4 & 8.24 & $\mathrm{HMC}(\mathrm{e} / \mathrm{o})$ & 0.120 & 0.98 \\
\hline $4 c$ & 4 & 7.21 & $\mathrm{HMC}(\mathrm{e} / \mathrm{o})$ & 0.200 & 0.93 \\
\hline
\end{tabular}

Table 1: Summary of the simulated parameter sets, which enter our performance studies of dynamical fermion algorithms. Quark masses are close to their critical values. 


\begin{tabular}{|c|c|c|c|c|c|}
\hline set & $g_{\mathrm{SF}}^{2}$ & $\tau_{\text {int }}$ & $t_{\text {update }} /[s]$ & $M_{\mathrm{cost}}$ & $D_{\text {cost }} / 10^{2}$ \\
\hline $12 \mathrm{a}$ & 1.1 & $2.00(16)$ & 1582 & $15(1)$ & $36(3)$ \\
\hline $12 \mathrm{~b}$ & 1.1 & $1.98(23)$ & 2392 & $20(2)$ & $30(4)$ \\
\hline $12 \mathrm{c}$ & 1.1 & $2.82(27)$ & 1773 & $22(2)$ & $37(4)$ \\
\hline $12 \mathrm{~d}$ & 1.1 & $1.41(10)$ & 1636 & $12.9(9)$ & $30(2)$ \\
\hline $12 \mathrm{e}$ & 1.1 & $1.35(7)$ & 1335 & $12.0(6)$ & $28(2)$ \\
\hline $12 f$ & 1.3 & $2.19(12)$ & 1768 & $15.0(8)$ & $38(2)$ \\
\hline $12 \mathrm{~g}$ & 1.3 & $1.16(7)$ & 1518 & $18.3(1.1)$ & $44(3)$ \\
\hline $12 \mathrm{~h}$ & 1.3 & $2.47(15)$ & 1103 & $11.6(7)$ & $27(2)$ \\
\hline $12 \mathrm{i}$ & 1.7 & $2.35(14)$ & 1895 & $14.7(9)$ & $39(2)$ \\
\hline $12 \mathrm{j}$ & 1.7 & $2.05(14)$ & 1554 & $12.6(9)$ & $32(2)$ \\
\hline $12 \mathrm{k}$ & 1.7 & $1.81(13)$ & 1371 & $11.6(9)$ & $28(2)$ \\
\hline $10 \mathrm{a}$ & 1.1 & $1.95(7)$ & 707 & $9.7(4)$ & $16.2(6)$ \\
\hline $10 b$ & 1.3 & $2.35(10)$ & 719 & $10.7(5)$ & $17.5(8)$ \\
\hline $10 \mathrm{c}$ & 1.7 & $2.63(13)$ & 804 & $11.5(6)$ & $18.7(9)$ \\
\hline $10 \mathrm{~d}$ & 2.1 & $3.42(20)$ & 806 & $14.4(8)$ & $25(1)$ \\
\hline $10 \mathrm{e}$ & 2.4 & $3.46(21)$ & 991 & $16(1)$ & $28(2)$ \\
\hline $10 \mathrm{f}$ & 3.3 & $3.42(21)$ & 1274 & $19(1)$ & $33(2)$ \\
\hline $10 \mathrm{~g}$ & 5.4 & $3.94(22)$ & 1932 & $30(2)$ & $49(3)$ \\
\hline $8 a$ & 1.1 & $1.14(4)$ & 260 & $4.0(1)$ & $8.2(3)$ \\
\hline $8 b$ & 1.3 & $1.40(6)$ & 183 & $3.0(1)$ & $6.2(3)$ \\
\hline $8 c$ & 1.7 & $2.40(9)$ & 239 & $5.8(2)$ & $8.4(3)$ \\
\hline $6 a$ & 1.0 & $0.70(2)$ & 49 & $1.00(3)$ & $1.98(6)$ \\
\hline $6 \mathrm{~b}$ & 1.1 & $0.75(2)$ & 49 & $1.04(3)$ & $2.09(6)$ \\
\hline $6 c$ & 1.2 & $0.80(2)$ & 55 & $1.15(3)$ & $2.34(6)$ \\
\hline $6 \mathrm{~d}$ & 1.5 & $1.08(2)$ & 74 & $2.08(4)$ & $4.50(8)$ \\
\hline $5 \mathrm{a}$ & 1.0 & $0.67(1)$ & 22 & $0.70(1)$ & $1.03(2)$ \\
\hline $5 b$ & 1.5 & $1.02(3)$ & 22 & $0.83(2)$ & $1.26(4)$ \\
\hline $4 a$ & 1.0 & $0.53(1)$ & 6 & $0.268(5)$ & $0.428(8)$ \\
\hline $4 \mathrm{~b}$ & 1.2 & $0.57(1)$ & 7 & $0.271(5)$ & $0.445(8)$ \\
\hline $4 c$ & 1.5 & $0.74(1)$ & 5 & $0.222(3)$ & $0.397(5)$ \\
\hline
\end{tabular}

Table 2: Cost estimates for the simulations in the parameter sets specified in Table 1 . Precise results and analysis on the running of $g_{\mathrm{SF}}^{2}$ will appear in 29. 


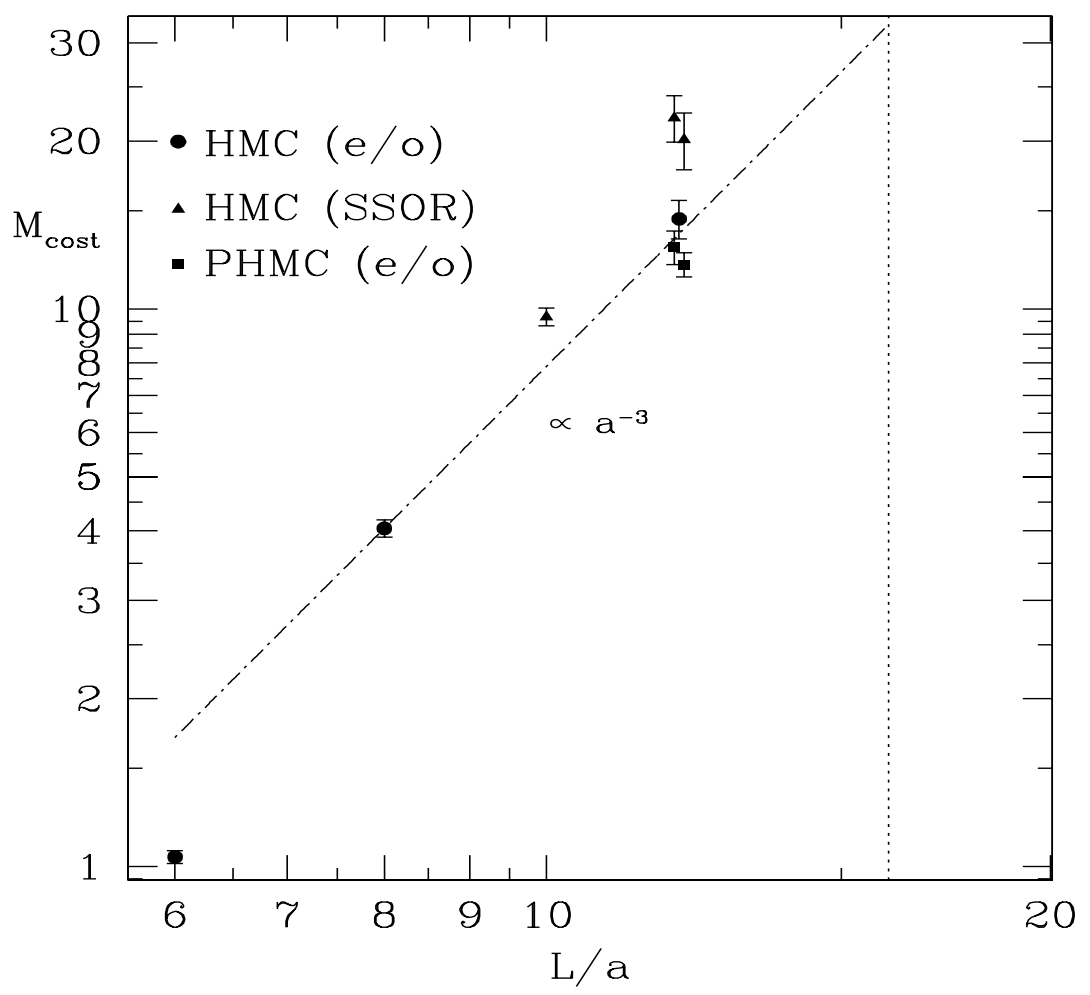

Figure 1: Measured values of $M_{\text {cost }}$ for runs with constant physics fixed by $g_{\mathrm{SF}}^{2} \approx 1.1$ and vanishing quark mass. 


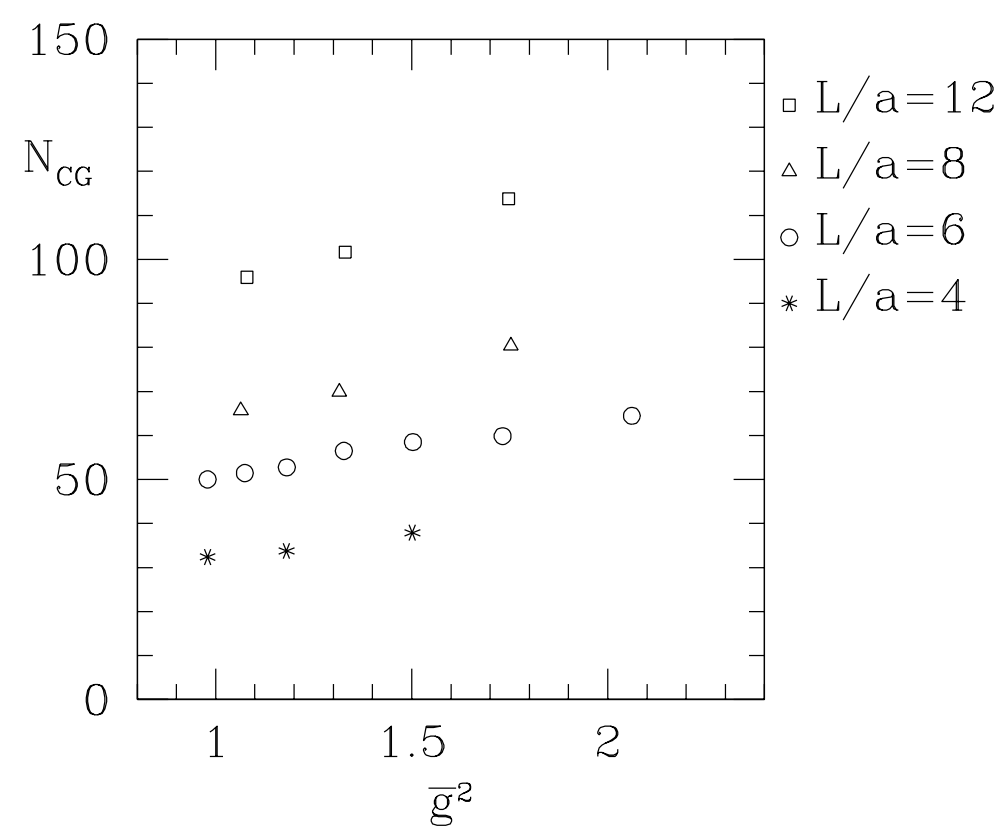

Figure 2: Average number of iterations of the conjugate gradient algorithm in even-odd preconditioned HMC runs.

\begin{tabular}{cccccccccccccc}
\hline$\beta$ & $r_{1}$ & $n_{1}$ & $n_{2}$ & $n_{3}$ & $n_{4}$ & $t_{1}$ & $t_{2}$ & $t_{3}$ & $t_{4}$ & $a_{1}$ & $a_{2}$ & $a_{3}$ & $a_{4}$ \\
\hline 8.39 & 4 & 15 & 31 & 63 & 255 & 1 & 6 & 6 & 10 & 0.313 & 0.828 & 0.966 & 0.998 \\
8.854 & 4 & 15 & 31 & 63 & 255 & 1 & 6 & 6 & 10 & 0.347 & 0.835 & 0.969 & 0.999 \\
9.40 & 3 & 11 & 23 & 63 & 255 & 1 & 8 & 10 & 10 & 0.355 & 0.742 & 0.923 & 0.996 \\
\hline
\end{tabular}

Table 3: Parameters of the MLM update cycle, where $l=4, r_{2}=r_{3}=r_{4}=1$ and $a_{j}$ denotes the acceptance rate at the level $j$. 


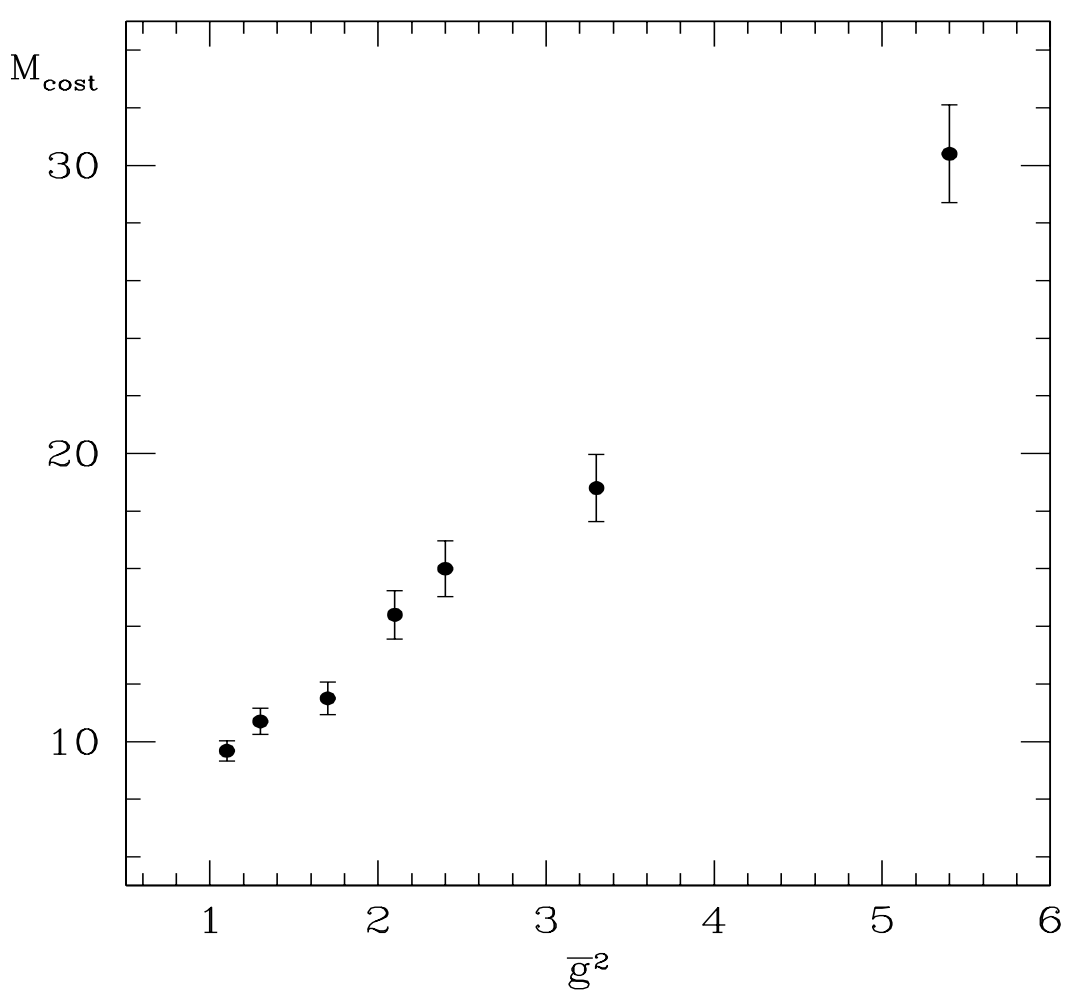

Figure 3: Measured values of $M_{\text {cost }}$ for runs with HMC-SSOR on $L / a=10$ lattices versus renormalized coupling.

\begin{tabular}{cccc}
\hline$\beta$ & 8.39 & 8.854 & 9.40 \\
\hline$g_{\mathrm{SF}}^{2}$ & $1.1807(12)$ & $1.0778(10)$ & $0.9767(16)$ \\
$D_{\text {cost }}$ & $290(6)$ & $287(6)$ & $372(15)$ \\
\hline
\end{tabular}

Table 4: $D_{\text {cost }}$ for simulations with the multi-level algorithm of $5^{4}$ lattices at various $\beta$-values. 


\begin{tabular}{cccccc}
\hline set & $\beta$ & $N_{\text {corr }}$ & $\epsilon$ & $n$ & $\delta$ \\
\hline $12 \mathrm{~d}$ & 9.5 & 4 & 0.0050 & 44 & 0.0034 \\
$12 \mathrm{e}$ & 9.5 & 2 & 0.0050 & 44 & 0.0034 \\
$12 \mathrm{~g}$ & 8.5 & 4 & 0.0044 & 44 & 0.0050 \\
$12 \mathrm{~h}$ & 8.5 & 2 & 0.0069 & 42 & 0.0016 \\
$12 \mathrm{j}$ & 7.5 & 4 & 0.0050 & 46 & 0.0026 \\
$12 \mathrm{k}$ & 7.5 & 2 & 0.0050 & 46 & 0.0026 \\
\hline
\end{tabular}

Table 5: Parameters controlling the polynomial approximation to $\hat{Q}^{-2}$ for PHMC runs on $12^{4}$.

\begin{tabular}{ccccc}
\hline set & $\left\langle\lambda_{\min }\right\rangle$ & $\left\langle\lambda_{\max }\right\rangle$ & $\tilde{c}_{0}$ & $\sigma_{N_{\text {corr }}}^{2} / \sigma_{P}^{2}$ \\
\hline $12 \mathrm{~d}$ & $0.002477(15)$ & $0.8582(1)$ & 0.6738653 & 1.05 \\
$12 \mathrm{e}$ & $0.002474(12)$ & $0.8582(1)$ & 0.6738653 & 1.10 \\
$12 \mathrm{~g}$ & $0.002284(14)$ & $0.8765(1)$ & 0.6686256 & 1.35 \\
$12 \mathrm{~h}$ & $0.002270(13)$ & $0.8765(1)$ & 0.6686256 & 1.14 \\
$12 \mathrm{j}$ & $0.001869(14)$ & $0.8664(1)$ & 0.6477127 & 1.06 \\
$12 \mathrm{k}$ & $0.001860(15)$ & $0.8667(1)$ & 0.6477127 & 1.03 \\
\hline
\end{tabular}

Table 6: Average smallest and largest eigenvalue of $\hat{Q}^{2}$ (see eq. (2.4) for $\tilde{c}_{0}$ ) and ratio of errors in eq. (B.2). 